\title{
15
}

\section{Accurate Approximation of the Cell Loss Ratio in ATM Buffers with Multiple Servers}

\section{B. Steyaert and H. Bruneel}

SMACS Research Group, Laboratory for Communications Engineering University of Ghent, Sint-Pietersnieuwstraat 41, B-9000 Gent, Belgium

In this paper, we focus attention on discrete-time buffer models with general independent arrivals and multiple output links, a class of queueing models which is well-suited to address performance issues in slotted systems, such as ATM. Calculating the Cell Loss Ratio (CLR), a key performance measure whenever finite-capacity buffers are involved, requires solving a set of linear equations, the size of which depends on the buffer capacity $K$. Therefore, the $C L R$ is often approximated by some appropriate tail probability of the buffer contents in the corresponding infinite-capacity queueing model; however, how these two quantities are related, is generally not very well known. In this paper, using a generating-functions approach, we establish an exact relation between the distribution of the number of cells lost per' slot in a finite-capacity queue, and the distribution of the buffer contents in the corresponding infinite-capacity queue. This eventually leads to an extremely accurate closed-form approximation for the $C L R$ in the finite-buffer system, that is easily evaluated.

\section{INTRODUCTION}

In ATM-based B-ISDN networks, a certain level of Quality of Service (QoS) is to be guaranteed to each individual user. This, among other things, includes imposing an upper bound on the admissible Cell Loss Ratio $(C L R)$, the value of which depends on the application involved. Consequently, having accurate tools to estimate the CLR becomes crucial when evaluating the overall performance of the network.

This work was supported by Alcatel Bell Telephone Mfg. Co. (Antwerp, Belgium), and by the Belgian National Fund for Scientific Research (NFWO). 
In this paper, we focus attention on discrete-time buffer models with general independent arrivals and multiple output links, a class of queueing models that naturally arises in the performance evaluation of ATM switching elements at various stages in the network (see e.g [1-9], and the references therein). As in most studies concerned with ATM-related discrete-time queueing models, the time axis is divided into slots of fixed length. The transmission of cells (units of information) is synchronized to the slot boundaries, and one slot suffices for the transmission of exactly one cell.

Let us consider a multiplexer model with $c(c \geq 1)$ transmission lines, implying that the number of cells that can leave the system during any slot is at most $c$. The multiplexer queue, where cells awaiting their transmission are temporarily stored, is assumed to have a storage capacity of $K$ cells; this does not include cells that are currently being transmitted, if any. In the following, we will denote by the random variable $a_{k}$ the number of new cell arrivals during slot $k$ attempting to enter the multiplexer buffer. The cell arrival model is general, and assumed to be i.i.d., meaning that during any slot, i.e., independently from cell arrivals during previous slots. Although, in general, ATM traffic tends to be of a bursty nature, it has been verified through simulation (e.g. [6]) that the i.i.d. assumption is quite realistic, for instance, in multistage switching networks where the arriving traffic is randomized (meaning that arriving cells are randomly distributed among all input links of the network). The number of new cell arrivals can then be characterized by a probability generating function $A(z)$, i.e.,

$A(z)=\mathrm{E}\left[z^{a} k\right]$

(where $\mathbf{E}[$.$] denotes the expected value of the argument), for any value of k$. The case where the numbers of cell arrivals during consecutive slots are correlated, requires a different approach, and is subject for further study. New cell arrivals are accepted as long as sufficient buffer space is available; otherwise, cells are dropped. Further, let us denote by $v_{k}$ the buffer contents at the beginning of slot $k$ (the total number of cells in the multiplexer buffer at this time instant, including those that will be transmitted during slot $k$, if any), i.e., observed just after possible cell departure epochs, but before new cell arrivals during this slot. Under these circumstances, $v_{k}$ cannot exceed $K$, and since, due to the synchronous cell transmission mode, cells that have entered the buffer during slot $k$ are still in the system at the beginning of slot $k+1, v_{k}$ and $v_{k+1}$ are related by the system equation 
$v_{k+1}=\min \left\{\left(v_{k}-c\right)^{+}+a_{k}, K\right\}$,

where $(.)^{+} \triangleq \max \{., 0\}$.

Calculating the CLR (also : Cell Loss Probability, Blocking Probability), defined as the fraction of new cells arrivals that are lost, due to the fact that the cell buffer of the system is completely filled at their arrival epoch, involves solving the $K+1$ balance equations (together with the normalization condition) defined by (1). Indeed, let us denote by $p c \triangleq A^{\prime}(1)$ (where primes denote derivatives with respect to the argument) the offered load, equal to the mean number of new cell arrivals per slot; from this definition it follows that $p$ is the mean number of cells offered per output link, per slot. Similarly, let us denote by the carried load the mean number of cells processed by the transmission unit per slot, which, in the steady-state, is equal to the mean number of cells per slot offered to the multiplexer buffer that can actually enter the system. Defining the steady-state probabilities

$q_{i} \triangleq \lim _{k \rightarrow \infty} \operatorname{Prob}\left[v_{k}=i\right] \quad, \quad s q_{i} \triangleq \lim _{k \rightarrow \infty} \operatorname{Prob}\left[v_{k} \leq i\right]$,

for all $0 \leq i \leq K$, the latter quantity can be calculated from

carried load $=\sum_{i=0}^{c-1} i q_{i}+\sum_{i=c}^{\infty} c q_{i}=c-\sum_{i=0}^{c-1}(c-i) q_{i}$.

The $C L R$, the fraction of cells that cannot enter the buffer, is then given by

$C L R=\frac{\text { offered load }- \text { carried load }}{\text { offered load }}=\sum_{i=0}^{c-1} \frac{c-i}{p c} q_{i}-\frac{1-p}{p}=\sum_{i=0}^{c-1} \frac{s q_{i}}{p c}-\frac{1-p}{p}$.

Calculating the values of $q_{i}$ (or, equivalently, $s q_{i}$ ), $0 \leq i \leq c-1$, requires solving the entire set of $K+1$ balance equations.

Solving the set of balance equations may be a complicated task, including substantial numerical difficulties and/or time consuming algorithms. On the other hand, under various circumstances, it has been observed that the $C L R$ in a finite queue and the distribution of the buffer occupancy in the corresponding infinite-capacity queue exhibit a similar asymptotic behavior when plotted versus the buffer size (or buffer contents) $K$. Therefore, in many cases, the $C L R$ is approximated by the latter quantity, because a number of techniques for approximating the distribution of the buffer occupancy exist, 
that have shown to be accurate in the range of values of interest (i.e., sufficiently small probabilities, see [7-13]). Nevertheless, in most cases, the exact correspondence between the number of cells lost per slot and the buffer occupancy in a queueing model with infinite storage capacity is not clear, and to the best of our knowledge, this issue has received little or no attention in the literature. In [14], a relation between the $C L R$ in the discrete-time $G e o^{[X]} / D / 1 / K$ queueing system, and the distribution of the buffer contents in the corresponding infinite-capacity queueing model was reported, which is a special case of the relationship to be derived in the current paper for the multiserver queueing model described above.

\section{GENERATING FUNCTION OF THE BUFFER CONTENTS}

In order to be able to derive an expression for the generating function of the buffer contents, let us denote by $\ell_{k}$ the number of cells lost during slot $k$, due to contention when new cells arrive at the multiplexer buffer which is already fully occupied. This random variable is related to $v_{k}$ and $a_{k}$ by the equation

$\ell_{k}=\left(\left(v_{k}-c\right)^{+}+a_{k}-K\right)^{+}$.

Then, defining $L_{k}(z)$ and $V_{k}(z)$ as the probability generating functions corresponding to the random variables $\ell_{k}$ and $v_{k}$ respectively,

$L_{k}(z) \triangleq \mathrm{E}\left[c_{z}^{\ell} k\right] \quad, \quad V_{k}(z) \triangleq \mathrm{E}\left[\begin{array}{c}v_{k} \\ z\end{array}\right]$,

the above relation can be translated to the z-domain as follows :

$L_{k}(z)=1+\mathrm{E}\left[z^{\left(v_{k}-c\right)^{+}+a_{k}-K}-1 \mid\left(v_{k}-c\right)^{+}+a_{k}>K\right] \operatorname{Prob}\left[\left(v_{k}-c\right)^{+}+a_{k}>K\right]$.

On the other hand, from system equation (1), we find that

$$
\begin{array}{r}
V_{k+1}(z)=\mathrm{E}\left[z_{z}^{\left.\left(v_{k}-c\right)^{+}+a_{k}\right]}+\mathrm{E}\left[z_{z-z}\left(v_{k}-c\right)^{+}+a_{k} \mid v_{k}-c\right)^{+}+a_{k}>K\right] \\
. P r o b\left[\left(v_{k}-c\right)^{+}+a_{k}>K\right] .
\end{array}
$$

Combining the two above equations, we obtain

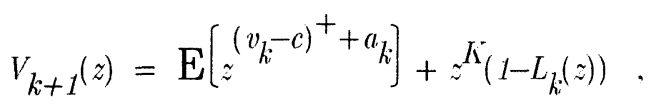


which can be further written as

$$
\begin{aligned}
& V_{k+1}(z)=z^{-c} A(z)\left(V_{k}(z)+(z-1) S Q_{k}(z)\right)+z^{K}\left(1-L_{k}(z)\right) \\
& S Q_{k}(z) \triangleq \sum_{i=0}^{c-1} \frac{z^{c}-z^{i}}{z-1} \operatorname{Prob}\left[v_{k}=i\right]=\sum_{i=0}^{c-1} \sum_{n=i}^{c-1} z^{n} \operatorname{Prob}\left[v_{k}=i\right]=\sum_{n=0}^{c-1} z^{n} \operatorname{Prob}\left[v_{k} \leq n\right] .
\end{aligned}
$$

The system reaches an equilibrium after a sufficiently large period of time, and the distribution of all random variables involved in the above analysis becomes independent of the exact value of $k$. Then, defining the steady-state functions

$$
V(z) \triangleq \lim _{k \rightarrow \infty} V_{k}(z) ; L(z) \triangleq \lim _{k \rightarrow \infty} L_{k}(z) ; S Q(z) \triangleq \lim _{k \rightarrow \infty} S Q_{k}(z)
$$

equation (4) leads to the following expression for the steady-state probability generating function of the buffer contents at the beginning of an arbitrary slot :

$$
V(z)=\frac{(z-1) A(z) S Q(z)+z^{K+c}(1-L(z))}{z^{c}-A(z)} .
$$

The buffer occupancy at the beginning of a slot is bounded by the value of $K$; therefore, the right-hand side in the above formula must be a polynomial in $z$ of degree $K$, implying that the denominator must be a divisor of the numerator. The latter property could be used to determine $V(z)$ from (5), but this would fall beyond the scope of this paper. Note that, since pc.CLR $=L^{\prime}(1)$, the mean number of cells lost per slot, the normalization condition $V(1)=1$ yields equation (2) for the $C L R$.

\section{INFINITE-BUFFER RESULTS}

The results for the infinite--capacity queueing model can also be derived from the above expression. In this case, the (infinite) set of balance equations will have a normalized solution, only if the equilibrium condition

$A^{\prime}(1)<c \Leftrightarrow p<1$.

is satisfied. Under these conditions, no cells will be lost, i.e., $L(z) \rightarrow 1$, as $K$ approaches infinity. Defining $U(z), p_{i}$ and $s p_{i}(0 \leq i \leq \infty)$ as the infinite-capacity limits of the probability generating function, distribution and cumulative distribution, respectively, of the buffer contents at the beginning of an arbitrary slot, we thus obtain 
$U(z)=\frac{(z-1) A(z) S P(z)}{z^{c}-A(z)}$,

where

$S P(z) \triangleq \sum_{i=0}^{c-1} z^{i} s p_{i}$

is still to be determined. The buffer occupancy can now take any value between 0 and infinity; therefore, we no longer have that the denominator in the right-hand side of (7.a) is a divisor of the numerator. Nevertheless, using Rouché's theorem (see e.g. [8]), it can be shown that the denominator $z^{c}-A(z)$ has $c-1$ zeros inside the unit disk $\{z$ : $|z|<1\}$. Then exploiting the property that $U(z)$, being a probability generating function, is analytic inside the unit disk, these must also be zeros of the denominator in the right-hand side of (7.a), a property which completely determines the (c-1)-th degree polynomial $S P(z)$. Indeed, denoting by $z_{j}, 1 \leq j \leq c-1$, the $c-1$ zeros of $z-A(z)$ satisfying $|z j|<1$, we find,

$S P(z)=c(1-p) \prod_{j=1}^{c-1} \frac{z-z j}{1-z_{j}}$,

where the normalization condition for $U(z)$ has been incorporated in (7.c). The $c$ unknown probabilities $s p$, $0 \leq i \leq c-1$, could be solved from (7.b,c) by identifying the coefficients of equal powers of $z$ in both expressions. However, in the following, we will derive an approximation for the $C L R$ such that the solution of this set of linear equations can be avoided. Expressions $(7 . a-c)$ for the probability generating function of the buffer contents are well-known results, and have been quoted in the literature on various occasions (e.g. see [6-8], [15-16]).

\section{AN EXPRESSION FOR $L(z)$}

In this section, we will establish a relationship between the $C L R$ and the buffer occupancy in an infinite-capacity buffer. For that purpose, we first derive an expression for $L(z)$, the probability generating function describing the number of cells lost per slot. Let us therefore combine equations (5) and (7.a) into

$$
S Q(z) U(z)-S P(z) V(z)=\frac{L(z)-1}{z^{c}-A(z)} z^{K+c} S P(z)
$$


If we take the $n$-th derivative of the above equation for $z=0$ (due to the probabilitygenerating property applied for the probability generating functions occurring in the above expression, these derivatives are well-defined and finite), we observe that the right-hand side will have non-zero contributions only if $n \geq K+c$. In other words, the coefficient of $z^{n}$ in the series expansion of the function $S Q(z) U(z)-S P(z) V(z)$ will be non-zero, only if $n \geq K+c$. Since $L(z)$ satisfies

$$
L(z)=1+\left(z^{c}-A(z)\right) \frac{S Q(z) U(z)-S P(z) V(z)}{z^{K+c} S P(z)}
$$

the latter property, together with the observation that $S P(z) V(z)$ is a polynomial in $z$ of degree $K+c-1$ (meaning that, considering the coefficients of $z^{n}, \mathrm{n} \geq K+c$, in the series expansion of the numerator in the right-hand side of this expression for $L(z)$, only $S Q(z) U(z)$ will have non-zero contributions), implies that $L(z)$, the probability generating function of the number of cells lost per slot, can be written as

$\left.L(z)=1+\left(z^{c}-A(z)\right) \cdot S P(z)-1 \sum_{\ell=1}^{c-1} s q_{\ell=c-\ell}^{c-1} \sum_{i=1}^{i+\ell-c} p_{K+i}+\frac{S Q(z)}{S P(z)} \sum_{i=K+c}^{\infty} z^{i-K-c} p_{i}\right\}$,

where we have introduced the appropriate series expansions for $S Q(z)$ and $U(z)$, in terms of the steady-state probabilities defined in the previous sections. From this expression for $L(z)$, all quantities of interest concerning the distribution of the number of cells lost per slot can be calculated. In this paper, we will limit ourselves to deriving the mean number of lost cells per slot. Specifically, taking the first-order derivative for $z=1$, and using the relations $S P(1)=c(1-p), S Q(1)=c(1-p)+L^{\prime}(1)$, and $L^{\prime}(1)=p c . C L R$, we find that the $C L R$ satisfies

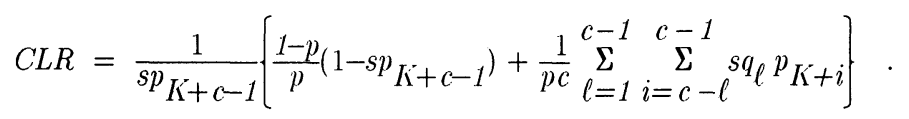

It is worth noting that the expression between braces in the right-hand side of this equation is a sum of nothing but positive terms. Therefore, introducing accurate approximations for some of these terms (as is done in the next section), will by no means have severe consequences on the final result. Also, in the single-server case $c=1$, this expression reduces to the following equation :

$$
C L R=\frac{1-p}{p} \frac{1-s p K}{s p} K
$$

which was established in [14] for the $G e o^{[X]} / D / 1 / K$ queueing system. 


\section{AN APPROXIMATION FOR THE $C L R$}

In this section, we derive an extremely accurate approximation for the $C L R$ in the multiserver case, by introducing some proper approximation for the quantities occurring in the right-hand side of (9). First of all, in [7], it was mentioned that extremely accurate data are obtained when using a geometric-tail approximation for the distribution of the buffer occupancy in the infinite-capacity queueing model, i.e,

$p_{i} \cong-C z_{0}^{-i-1}$, for large $i$,

which, upon suitable summation, also yields

$$
1-s p_{i} \cong-C \frac{z_{0}^{-i-1}}{z_{0}-1}
$$

In (11.a,b), $z_{0}$ is the zero outside the unit disk of $z^{c}-A(z)$ (the denominator in expression (7.a) for $\mathrm{U}(\mathrm{z})$ ) with the smallest modulus, a real and positive quantity larger than 1 , and, from the residue theorem, $C$ is given by

$$
C=\lim _{z \rightarrow z_{0}}\left(z-z_{0}\right) U(z)=\frac{\left(z_{0}-1\right) A\left(z_{0}\right) S P\left(z_{0}\right)}{c z_{0}{ }^{-1}-A^{\prime}\left(z_{0}\right)}
$$

The accuracy of the above approximations becomes better as $i$ increases, and is already very good even for relatively small values of $i$. It is self-evident to use approximation $(11 . \mathrm{a}-\mathrm{c})$ for $1-\mathrm{s} p_{\mathrm{i}}$ and $p_{i}$, the (negative cumulative) distribution of the buffer contents in an infinite-capacity buffer, in expression (9) for the $C L R$.

On the other hand, $s q_{p}, 1 \leq \ell \leq c-1$, the cumulative distribution of the buffer contents in a buffer of size $K$, can only be calculated by solving the set of balance equations, which, of course, we wish to avoid. Therefore, note that, when the CLR is sufficiently small, the difference between $s q_{\ell}$ and $s p_{\ell}, 1 \leq \ell \leq c-1$, will be marginal, and the latter quantities, which could be calculated from $(7 . b, c)$, can be used as an approximation for the $s q_{\rho}$ 's. Then it is not difficult to show that the second term between braces in the right-hand side of (9) can be written as

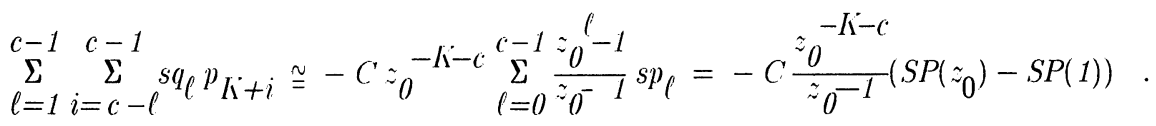


Combining the latter expression, equation (7.c) for $S P(z)$, and approximation (11.b) with the expression between braces in the right-hand side of (9), we thus find

$C L R \cong-C \frac{1-p}{p} \frac{z^{-K-c}}{z-1} \prod_{j=1}^{c-1} \frac{0^{-z} j}{1-0_{j}}$,

under the assumption that $s p_{K+c-1} \cong 1$ in the denominator of (9), which will be the case if the parameters are such that the $C L R$ is sufficiently small. In the next section, through some numerical examples, we will verify that (12) forms a close approximation for the exact value of the $C L R$. The calculation of the right-hand side in (12) involves calculating the zeros inside the unit disk, and the zero outside the unit disk with the smallest modulus of $z^{c}-A(z)$, a simple numerical task that can be executed without difficulties using a Newton-Raphson iteration scheme.

\section{NUMERICAL EXAMPLES}

Let us consider an ATM switching element with $N$ input links and $N$ output links and output buffering $([4-8])$. Depending on their destination, the output links are grouped together, which gives rise to $N / c$ possible destination groups, each having $c$ output links for the transmission of cells. On each input link, the cell arrival process is a Bernoulli process with mean $p$, i.e., during any slot, there is either 1 cell arrival (probability $p$ ) or no cell arrival at all (probability $1-p$ ), independent from slot to slot. Cell arrival processes on different input links are independent. The routing of cells to their respective destinations is uniform and independent, meaning that each output group has probability $c / N$ of being addressed by an arriving cell. One output buffer is provided per output group, each having storage capacity $K$. Under these circumstances, the aggregate cell arrival process per slot in an output buffer is a binomial process, the associated probability generating function being given by

$A(z)=\left(1+\frac{p c}{N}(z-1)\right)^{N}$.

The discrete-time multiserver queueing model we thus obtain for any of the output buffers has been described in Section 1, and the analysis from the previous sections can be applied, resulting in equation (12) as an approximation for the CLR in an output buffer. In Fig. 1, we have plotted the exact value for the CLR (marks) and the approximation obtained with (12) (full line) versus the buffer size $K$, for $N=16, c=4$, $p=0.2,0.4,0.6,0.8$, respectively. Calculating the exact values for the $C L R$ requires solving a set of $K+1$ balance equations (together with the normalization condition) for 
each individual value of $K$, while, on the other hand, the approximate results are readily obtained from (12), regardless the value of $K$, once the zeros $z ;, 1 \leq j \leq c-1$, and $z$, have been calculated. From Fig. 1, it is observed that the approximation becomes extremely accurate, even for relatively small values of $K$.

As $N$ becomes larger and larger, the maximum number of cells that may enter an output buffer during each slot increases, and for $N \rightarrow \infty$, the aggregate arrival process in an output queue becomes a Poisson process with mean $p c$, i.e.,

$\mathrm{A}(z)=\exp \{p c(z-1)\}$

which is often used as a worst-case model for the binomial arrival process (13.a). In Fig. 2, we have plotted the exact value for $C L R$ (marks) and the corresponding approximation (full line) versus the buffer size $K$, for $c=4, p=0.2,0.4,0.6,0.8$, respectively. Again, the approximation proposed in the previous section, which is easy to evaluate, leads to extremely accurate results, especially in the probability region of interest for ATM (i.e., $C L R<<1 \mathrm{E}-4$ ).

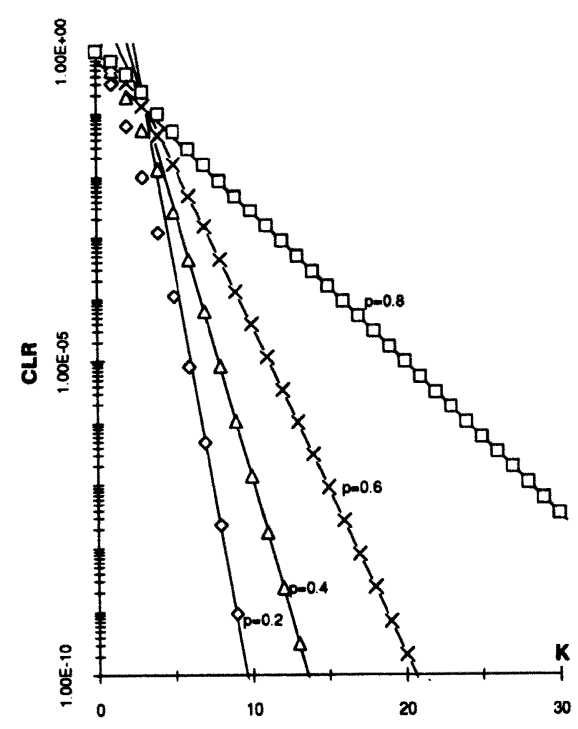

FIG. 1 Exact (marks) and approximate (full) CLR versus $K$, binomial arrival process, $c=4, N=16, p=0.2,0.4,0.6,0.8$.

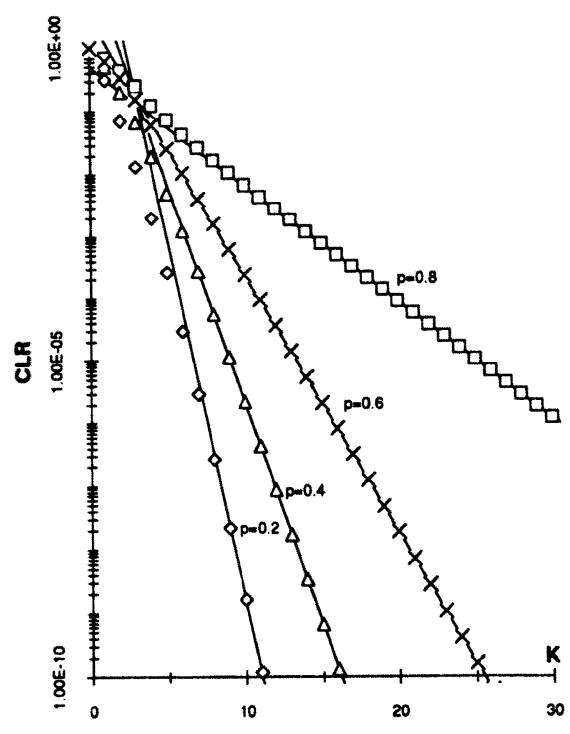

FIG. 2 Exact (marks) and approximate (full) CLR versus $K$, Poisson arrival process, $c=4, p=0.2,0.4,0.6,0.8$. 


\section{CONCLUSIONS}

In this paper, we have addressed the problem of deriving results for quantities which are typical for buffers with finite storage capacity (such as the CLR, a performance measure of considerable interest in future B-ISDN networks based on ATM) from infinite-buffer results, for, in general, the latter quantities are easier to obtain. In all of our derivations, we have used a generating-function approach, which has proven to be very useful, even when finite-capacity buffers are involved. Specifically, for a discrete-time multiserver queueing model with general independent arrivals, we have established an exact relationship between the probability generating function of the number of cells lost per slot in a buffer of capacity $K$, and the distribution of the buffer contents in a buffer with infinite storage capacity. This, in turn, has led to a closed-form approximate formula for the cell loss ratio, a quantity proportional to the mean number of cells lost per slot. Based on some numerical examples, we have shown that this approximation is extremely accurate, especially for those values for the $C L R$ of interest in the ATM framework.

\section{REFERENCES}

[1] O.H. Elmahgiubi and H.T. Mouftah, "Performance analysis of output buffered multipath multistage interconnection networks", Proceedings of ICC'93 (Geneva, 1993), pp. $1686-1690$.

[2] E. Del Re and R. Fantacci, "Performance evaluation of input and output queueing techniques in ATM-switching systems", IEEE Trans. Commun., vol. COM-41 (1993), pp. 1565-1575.

[3] A. Guerrero and F. Lozano, "Queueing models applying to a class of multiplexers", Proceedings of ITC 13 (Copenhagen, 1993), vol. Teletraffic and Datatraffic in a Period of Change, pp. 999-1004.

[4] M.J. Karol, M.G. Hluchyj and S.P. Morgan, "Input versus output queueing on a space-division packet switch", IEEE Trans. Commun., vol. COM-35 (1987), pp. $1347-1356$.

[5] Y. Oie, M. Murata, K. Kubota and H. Miyahara, "Performance analysis of nonblocking packet switch with input and output buffers", IEEE Trans. Commun., vol. COM-40 (1992), pp. 1294-1297. 
[6] G.H. Petit, A. Buchheister, A. Guerrero and P. Parmentier, "Performance evaluation methods applicable to an ATM multi-path self-routing switching network", Proceedings of ITC 13 (Copenhagen, 1991), vol. Teletraffic and Datatraffic in a Period of Change, pp. 917-922

[7] E. Desmet, B. Steyaert, H. Bruneel and G.H. Petit, "Tail distribution of queue length and delay in discrete-time queueing models, applicable in ATM networks", Proceedings of ITC 13 (Copenhagen, 1991), vol. Queueing, Performance and Control in ATM, pp. 1-6.

[8] H. Bruneel and B.G. Kim, Discrete-Time Models for Communication Systems Including ATM (Kluwer Academic Publishers, Boston, 1993).

[9] G.W.F. Fredrikson, "Buffer behavior for binomial input and constant service", IEEE Trans. Commun., vol. COM-22 (1974), pp. 1862-1866.

[10] B. Steyaert and H. Bruneel, "Analysis of ATM switching modules with channel grouping in a bursty-source environment", Proceedings of IBCN\&S (Copenhagen, 1993), pp. 383-394.

[11] K. Sohraby, "Highly-bursty sources and their admission control in ATM networks", Asynchronous Transfer Mode Networks (Plenum Press, New York, 1993), pp. 123-133.

[12] J.N. Daigle, Y. Lee and M.N. Magelhães, "Discrete-time queues with phase-dependent arrivals", Proceedings of INFOCOM'90 (San Francisco, CA., 1993), pp. $728-732$.

[13] Z. Zhang and A.S. Acampora, "Effect of on/off distributions on the cell loss probability in ATM networks", Proceedings of GLOBECOM'92 (Orlando, FA., 1992), pp. 1533-1539.

[14] C. Bisdikian, J.S. Lew and A.N. Tantawi, "On the tail approximation of the blocking probability of single server queues with finite buffer capacity", Queueing Networks with Finite Capacity (Elsevier Science Publishers, Amsterdam, 1993), pp. $267-280$.

[15] I. Rubin, "Access-control disciplines for multi-access communication channels : reservation and TDMA schemes", IEEE Trans. Inform. Theory, vol. IT-25 (1979), pp. 516-536.

[16] H.W. Lee and L. Liang, "A generalized analysis of message delay in STDMA", Comp. Netw. and ISDN Syst., vol. 19 (1990), pp. 11-24. 